\title{
On the vacuum stability of SUSY models
}

\author{
José Eliel Camargo-Molina*, ${ }^{*}$ Ben O’Leary, ${ }^{a}$ Werner Porod ${ }^{a}$ and Florian Staub ${ }^{b}$ \\ ${ }^{a}$ Institut für Theoretische Physik und Astronomie, Universität Würzburg \\ Am Hubland, 97074 Würzburg, Germany \\ ${ }^{b}$ Bethe Center for Theoretical Physics \& Physikalisches Institut der Universität Bonn, \\ 53115 Bonn, Germany \\ E-mail: jose.camargo@physik. uni-wuerzburg.de, \\ ben.oleary@physik.uni-wuerzburg.de, porodephysik.uni-wuerzburg.de, \\ fnstaub@th.physik.uni-bonn.de
}

The existence of multiple non-equivalent minima of the scalar potential in SUSY models both raises technical challenges and introduces interesting physics. The technical challenges are now that one has to find several minima and evaluate which is the deepest, as well as calculate the tunneling time from a false vacuum to the true vacuum. We present here studies on the vacuum stability and color/charge breaking minima in the CMSSM and $R$ parity violating minima in a B-L extended MSSM.

The European Physical Society Conference on High Energy Physics 18-24 July, 2013

Stockholm, Sweden

${ }^{*}$ Speaker. 


\section{Introduction}

An important part of the phenomenology of the incredibly successful standard model of particle physics (SM) is the spontaneous breaking of some (but not all) of the gauge symmetries of the Lagrangian density by the vacuum expectation value (VEV) of a scalar field charged under a subgroup of the SM gauge group. The entire scalar sector of the SM consists of a doublet of $S U(2)_{L}$ with $-\frac{1}{2} U(1)_{Y}$ hypercharge. The potential energy of the vacuum is minimized by the scalar field taking a constant non-zero value everywhere. The presence of this VEV allows for massive particles that would be forced to be massless if the gauge symmetries of the Lagrangian density were also symmetries of the vacuum state.

Many extensions of the SM introduce extra scalar fields. Sometimes these fields are introduced explicitly to spontaneously break an extended gauge symmetry down to the SM gauge group e.g.[1, 2], and they are assumed to have non-zero VEVs at the true vacuum of the theory. Other times they are introduced for other reasons, such as supersymmetry [3], and often non-zero VEVs for such fields would be disastrous, such as breaking $S U(3)_{c}$ and/or $U(1)_{\mathrm{EM}}$, which excludes certain parts of the parameter space of the minimal supersymmetric standard model (MSSM) from being phenomenologically relevant. The existence of multiple non-equivalent vacua both raises technical challenges and introduces interesting physics. The technical challenges are now that one has to find several minima and evaluate which is the deepest, as well as calculate the tunneling time from a false vacuum to the true vacuum.

The technical challenges are much tougher when multiple scalar fields are involved. Even a tree-level analysis involves solving a set of coupled cubic equations, the so-called minimization or tadpole equations. It has generally only been attempted for highly symmetric systems such as two Higgs doublet models $[4,5]$ or with only a minimal amount of extra degrees of freedom such as the (assumed) three non-zero VEVs of the next-to-minimal supersymmetric standard model $[6,7,8]$.

The program Vevacious [9] has been written to address this. Given a set of tadpole equations and the terms needed to construct the one-loop effective potential, Vevacious brings together a series of public tools to find the minima of one-loop potentials and if required calculate the tunneling time between them.

We present here highlights from studies performed on the vacuum stability of two supersymmetric models. In section 2 we showcase some results from [10], where we study the color and charge breaking minima that might appear in the mSUGRA inspired Constrained MSSM (CMSSM). In section 3 we discuss some results from [11], where we investigate the issue of $R$ parity violation in the B-L extended CMSSM, as for this model $R$ parity can be violated spontaneously through VEVs for the scalar partners of right-handed neutrinos.

\section{Revisiting the vacuum structure of the CMSSM}

\subsection{The model}

One popular way of ameliorating the standard model hierarchy problem is to promote it to a supersymmetric theory, such as the MSSM [12]. The mechanism of supersymmetry (SUSY) breaking introduces more parameters and the breaking of SUSY is often parametrized by adding soft SUSY-breaking terms to the Lagrangian density. The number of parameters specifying the 


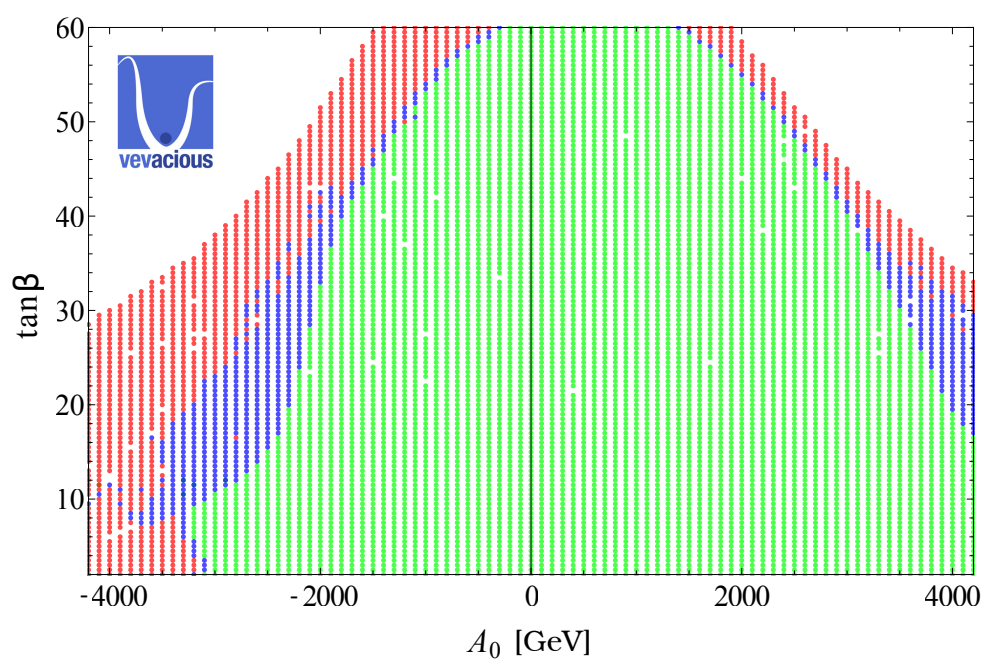

Figure 1: Projection in the $\tan \beta / A_{0}$ plane for a random scan in the CMSSM $\left(M_{0}\right.$ and $M_{1 / 2}$ are fixed at 1 $\mathrm{TeV}$ ). Green points have the input color- and charge- conserving minimum as global minimum. Blue and red points have charge- and color- breaking minima which are deeper than the input minimum. Red points have tunneling times from the input minimum to the CCB minimum lower than one tenth of the age of the Universe. Blue points have a tunneling time greater than a tenth of the age of the Universe.

full set of soft SUSY-breaking terms allowed in the MSSM is rather large, namely 105, so often they are taken to be related at a specific scale. One of the simplest and most popular proposal is the minimal-supergravity-inspired constrained MSSM (CMSSM), in which all the soft SUSYbreaking scalar mass-squared terms and the mass terms for the fermionic partners of the gauge bosons are taken to be equal to $M_{0}^{2}$ and $M_{1 / 2}$ respectively at the scale $\left(M_{G U T}\right)$ where the gauge couplings unify. The $A$ terms, which multiplied by the corresponding Yukawa couplings give the trilinear scalar interaction couplings, are also taken to unify at the value $A_{0} .|\mu|$ and $B_{\mu}$ are fixed by requiring that the mass of the $Z$ boson is correct along with defining the ratio $\tan \beta$ of the VEVs $v_{d}$ and $v_{u}$ of the neutral components of the two Higgs doublets, and the sign of $\mu$ is given as a final input.

The presence of many additional scalar partners for the SM fermions raises the question of whether they too could develop VEVs. Unfortunately, until recently it was quite impractical to search for other vacua to see whether the desired vacuum is stable, or whether there are chargeand/or color-breaking (CCB) minima.

We use Vevacious to investigate regions of the CMSSM which, despite having local minima with the desired breaking of $S U(2)_{L} \times U(1)_{Y}$ to $U(1)_{E M}$ while preserving $S U(3)_{c}$, might have global minima with a different breaking of the gauge symmetries.

\subsection{Results}

In fig. 1 we present results from a random scan in the $A_{0} / \tan \beta$ plane where the GUT-scale parameters $M_{0}$ and $M_{1 / 2}$ were fixed at $1 \mathrm{TeV}$ and $\mu$ was taken to be positive.

In this particular plot it is interesting to see that a large "green area" remains where the input minimum is the global one. However, a considerable amount of points develop stop and stau VEVs 
in areas of the parameter space that are not otherwise excluded by experiment. This shows the potential use of vacuum stability and the appearance of color- and charge- breaking minima as a phenomenological constraint. It is worth noting that for $\tan \beta<10$ we encountered points with mostly stop VEVs reflecting that enhancement of $Y_{t}$ might play a significant role, whether for high $\tan \beta$ stau VEVs were dominant as now $Y_{\tau}$ and $A_{\tau}$ are significantly enhanced, thus making it easier for stau VEVs to develop. It is interesting to point out that to obtain an LSP below $1 \mathrm{TeV}$ (important for Dark Matter studies), light staus are needed. This in turn implies large values of $\left|A_{0}\right|$ and/or $\tan \beta$. As can be seen in fig.1, this is a dangerous area as very often we get stau VEVs.

\section{Stability of $R$ parity in the BLSSM}

\subsection{The model}

There are several ways to extend the MSSM by $U(1)_{B-L}$. For this study, we confine ourselves to the minimal of such extensions, which allows for a spontaneously broken $U(1)_{B-L}$ without necessarily breaking $R$ parity. This requires the addition of two SM gauge-singlet chiral superfields $(\eta, \tilde{\eta})$ carrying $B-L$ charge which have to develop VEVs for reasonable phenomenological results, as well as the addition of three generations of superfields containing right-handed neutrinos. Analogous to the CMSSM, we reduce the number of free parameters by assuming unification of the gaugino masses, the soft SUSY-breaking scalar squared masses and trilinear couplings. We refer to this model as the BLSSM.

The BLSSM has a rich phenomenology, with a $Z^{\prime}$ boson [13], Majorana neutrinos with seesaw masses, several qualitatively new dark matter candidates [14] and a rich Higgs sector [15]. However most of the phenomenological studies for the BLSSM assume that $R$ parity is conserved.

The main idea behind investigating the vacuum structure of the BLSSM is to get a picture of how robustly $R$ parity is conserved for parameters of phenomenological interest, as $U(1)_{B-L}$ might be spontaneously broken by VEVs for the scalar partners of the right handed neutrinos (R-sneutrinos) instead of the being broken by $\eta$ and $\tilde{\eta}$ fields thus generating $R$ parity-violating interactions.

$\eta$ is responsible for generating a Majorana mass term for the right-handed neutrinos and thus we interpret the $B-L$ charge of this field as its lepton number, and likewise for $\bar{\eta}$, and call these fields bileptons since they carry twice the lepton number of (anti-)neutrinos. We summarize the quantum numbers of the extra chiral superfields with respect to the model's gauge group $U(1)_{Y} \times$ $S U(2)_{L} \times S U(3)_{C} \times U(1)_{B-L}$ in Tab. 1.

\begin{tabular}{|c|c|c|c|c|}
\hline \hline Superfield & Spin 0 & Spin $\frac{1}{2}$ & Generations & $U(1)_{Y} \otimes S U(2)_{L} \otimes S U(3)_{C} \otimes U(1)_{B-L}$ \\
\hline$\hat{v}^{c}$ & $\tilde{v}^{c}$ & $v^{c}$ & 3 & $\left(0, \mathbf{1}, \mathbf{1}, \frac{1}{2}\right)$ \\
$\hat{\eta}$ & $\eta$ & $\tilde{\eta}$ & 1 & $(0, \mathbf{1}, \mathbf{1},-1)$ \\
$\hat{\bar{\eta}}$ & $\bar{\eta}$ & $\tilde{\bar{\eta}}$ & 1 & $(0, \mathbf{1}, \mathbf{1}, 1)$ \\
\hline \hline
\end{tabular}

Table 1: Extra chiral superfields, in addition to those of the MSSM, and their quantum numbers. 

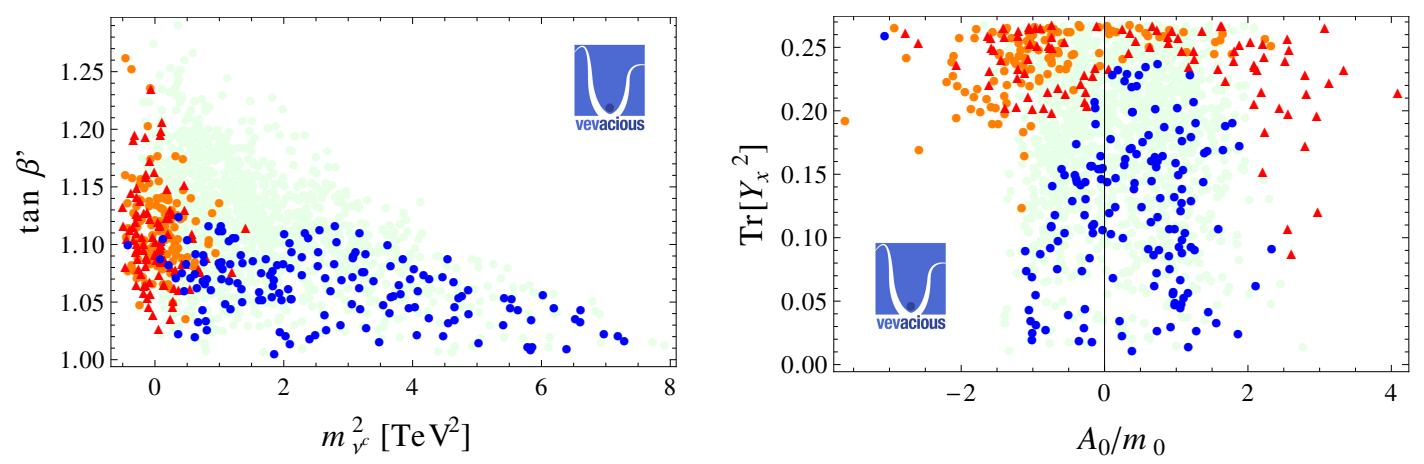

Figure 2: Projections onto various parameter planes of the 1640 hierarchical scan parameter points, categorized by the nature of their global minima at the one-loop level. $m_{v^{c}}^{2}$ denotes the smallest soft SUSY-breaking mass-squared for sneutrinos. "RPC" points are plotted in green, "RPV" points are in two groups based on the tunneling time from the "RPC" input minimum to the deeper "RPV" minimum: lower than one tenth of the age of the Universe as orange circles, greater than a tenth of the age of the Universe as red triangles. "Gauge conserving" points are in blue.

The superpotential is given by

$$
\begin{aligned}
W= & Y_{u}^{i j} \hat{u}_{i}^{c} \hat{Q}_{j} \hat{H}_{u}-Y_{d}^{i j} \hat{d}_{i}^{c} \hat{Q}_{j} \hat{H}_{d}-Y_{e}^{i j} \hat{e}_{i}^{c} \hat{L}_{j} \hat{H}_{d}+\mu \hat{H}_{u} \hat{H}_{d} \\
& +Y_{v}^{i j} \hat{v}_{i}^{c} \hat{L}_{j} \hat{H}_{u}-\mu^{\prime} \hat{\eta} \hat{\bar{\eta}}+Y_{x}^{i j} \hat{v}_{i}^{c} \hat{\eta} \hat{v}_{j}^{c},
\end{aligned}
$$

with the corresponding soft SUSY-breaking terms.

We denote the VEVs for the bilepton fields as $v_{\eta}$ and $v_{\bar{\eta}}$, for the sneutrinos of the $S U(2)_{L}$ doublets $\tilde{L}_{i}$ by $v_{L, i}$ and those of the $S U(2)_{L}$ singlet sneutrinos $\tilde{v}_{i}^{c}$ by $v_{R, i}$, with $i=1,2,3$.

\subsection{Results}

Two scans were performed for this model. The first scan, which we refer to as the "democratic" scan, took random values for each diagonal entry of the R-sneutrino - bilepton Yukawa coupling $Y_{x}$ independently over its range. The other, which we refer to as the "hierarchical" scan, kept the $(1,1)$ and $(2,2)$ entries as $10^{-3}$ and $10^{-2}$ respectively. We classify the stability results in 3 categories. The "RPC" category includes points for which the input $R$ parity-conserving minimum is the global minimum, the "RPV" category includes points for which a $R$ parity-violating minimum was found to be the deepest minimum. The "unbroken" category includes points that broke $S U(2)_{L}$ without breaking $U(1)_{B-L}$. Not all parameter points that are "RPC" at the one-loop level were "RPC" at tree level, and likewise for the "RPV" category. We present a summary of the results In table 2.

\begin{tabular}{|c|c|c|c|c|}
\hline Categorization & \multicolumn{2}{|c|}{ Hierarchical scan } & \multicolumn{2}{c|}{ Democratic scan } \\
\hline total & \multicolumn{2}{|c|}{1640} & \multicolumn{2}{c|}{2330} \\
\hline & tree level & one-loop level & tree level & one-loop level \\
\hline "RPC" & 1422 & 1275 & 2236 & 2167 \\
\hline "RPV" & 218 & 212 & 94 & 86 \\
\hline "unbroken" & 0 & 153 & 0 & 77 \\
\hline
\end{tabular}

Table 2: Number of parameter points in the various categories. 
In fig. 2 , we present these results plotted in some cuts of parameter space, including as well the result from the tunneling time calculation. There it is evident that smaller (and negative) sneutrino soft SUSY-breaking masses-squared are more likely to lead to smaller sneutrino masses and trigger VEVs for the sneutrinos, breaking $R$ parity. Also higher values for $Y_{X}$ play an important role, as $R$ parity is violated more often for higher values of $\operatorname{Tr}\left[Y_{X}^{2}\right]$. This is because the potential has destabilizing terms such as $-v_{R}^{2} v_{\bar{\eta}} Y_{X} \mu^{\prime}$. However, we would like to point out that these are only trends and for example, $R$ parity-conserving points are found for negative soft SUSY-breaking sneutrino masses-squared and vice versa, which slightly disagrees with previous results in literature [16].

\section{Conclusions}

When dealing with models that have extra scalar fields and extended Higgs sectors, understanding the vacuum structure becomes crucial for phenomenological studies. The common approach of choosing free parameters to satisfy the minimization conditions for a desired set of VEVs leaves out a plethora of minima that can be unphysical and lower than the desired one. The power of the homotopy continuation method for finding tree-level minima combined with gradient-based minimization with loop corrections has been combined in the publicly-available code Vevacious [9]. We used this tool to investigate the vacuum structure of two interesting supersymmetric models and presented some of the results. We showed that finding the global minimum of the one-loop effective potential sheds some light in phenomenological issues like spontaneous breaking of $R$ parity in the BLSSM and the appearance of charge- and color- breaking minima in the CMSSM. The current implementation of this procedure allows for the quick analysis of broad parameter scans, therefore making it feasible to be included as an extra constraint for the exclusion of regions in parameter space.

\section{References}

[1] P. Langacker, The Physics of Heavy Z' Gauge Bosons, Rev.Mod.Phys. 81 (2009) 1199-1228, [arXiv:0801.1345].

[2] L. Basso, S. Moretti, and G. M. Pruna, The Higgs sector of the minimal B-L model at future Linear Colliders, Eur. Phys. J. C71 (2011) 1724, [arXiv: 1012 . 0167].

[3] H. P. Nilles, Supersymmetry, Supergravity and Particle Physics, Phys.Rept. 110 (1984) 1-162.

[4] T. Lee, A Theory of Spontaneous T Violation, Phys.Rev. D8 (1973) 1226-1239.

[5] G. Branco, P. Ferreira, L. Lavoura, M. Rebelo, M. Sher, et al., Theory and phenomenology of two-Higgs-doublet models, Phys.Rept. 516 (2012) 1-102, [arXiv:1106.0034].

[6] P. Fayet, Supergauge Invariant Extension of the Higgs Mechanism and a Model for the electron and Its Neutrino, Nucl.Phys. B90 (1975) 104-124.

[7] J. R. Ellis, J. Gunion, H. E. Haber, L. Roszkowski, and F. Zwirner, Higgs Bosons in a Nonminimal Supersymmetric Model, Phys.Rev. D39 (1989) 844.

[8] M. Drees, Supersymmetric Models with Extended Higgs Sector, Int.J.Mod.Phys. A4 (1989) 3635. 
[9] J. Camargo-Molina, B. O’Leary, W. Porod, and F. Staub, Vevacious: A Tool For Finding The Global Minima Of One-Loop Effective Potentials With Many Scalars, arXiv:1307.1477.

[10] J. Camargo-Molina, B. O'Leary, W. Porod, and F. Staub, Stability of the CMSSM against sfermion VEVs, arXiv:1309.7212.

[11] J. Camargo-Molina, B. O'Leary, W. Porod, and F. Staub, The Stability Of R-Parity In Supersymmetric Models Extended By $U(1)_{B-L}$, Phys.Rev. D88 (2013) 015033, [arXiv: 1212 . 4146].

[12] S. P. Martin, A Supersymmetry primer, hep-ph/9709356.

[13] M. E. Krauss, B. O'Leary, W. Porod, and F. Staub, Implications of gauge kinetic mixing on Z' and slepton production at the LHC, Phys.Rev. D86 (2012) 055017, [arXiv: 1206 . 3513].

[14] L. Basso, B. O'Leary, W. Porod, and F. Staub, Dark matter scenarios in the minimal SUSY B-L model, JHEP 1209 (2012) 054, [arXiv: 1207 . 0507].

[15] L. Basso and F. Staub, Enhancing $h \rightarrow \gamma \gamma$ with staus in SUSY models with extended gauge sector, arXiv:1210.7946.

[16] P. Fileviez Perez and S. Spinner, The Fate of R-Parity, Phys. Rev. D83 (2011) 035004, [arXiv:1005.4930]. 